Methods Notes of 20 patients who died in Aneurin Bevan Health Board during the COVID-19 pandemic were audited for discussions of PPD and TEPs.

Results Even in cases where patients remained stable after the decision for supportive care was made, only in one case was PPD discussion documented. In contrast, in almost all cases there was a DNAR in place that was also discussed with the patient and/or family.

Conclusions Discussing TEPs is not necessarily the equivalent of robust joint decision-making. As we continue to manage dying patients during the pandemic, factors influencing PPD discussions in view of the wider context of end-of-life discussions should be considered. There is scope for research into how the COVID-19 pandemic has influenced or changed the quality of end-of-life discussions in secondary care to ensure patient-centred care moving forward.

\section{REMEMBER ME: SUPPORT THROUGH REMEMBRANCE DURING THE COVID19 PANDEMIC}

Ruth Pryce, Rachel Morris, Sarah Stanley. Marie Curie Hospice Liverpool

10.1136/spcare-2021-PCC.48

Background The COVID19 pandemic has highlighted a greater need for spiritual support and remembrance in a difficult time when usual comforts associated with end of life care have been stripped away. Marie Curie Hospice Liverpool have worked to develop unique ways to continue support through spiritual care and remembrance using technology and links to our local communities.

Methods There have been many challenges throughout the pandemic including the closure of the hospice to visitors, meaning for patients that face to face contact with relatives or their own faith leaders was no longer possible. Our chaplain continues to provide spiritual care for patients and carers, whilst creating remembrance opportunities. Embracing technology and the support of the local community has been vital in enabling patients to receive the best possible spiritual care. An example of this is the engagement of local church groups supporting the hospice by providing knitted hearts to keep patients and relatives connected. Remembrance opportunities have been created, including: online memorial services, use of technology to allow patients and families to come together for prayer, weekly email reflections to support staff wellbeing, and developing the chapel into an 'escape room' providing space for reflection during difficult times.

Results Results are positive. Patients and carers report feeling supported spiritually in a difficult time, which has been essential to their care. Remembrance work via online platforms has allowed our hospice and local community to be supported through bereavement and feedback from carers, local community groups and staff has been positive and complimentary.

Conclusions Our work has demonstrated that despite the challenges presented during the pandemic, spiritual care and remembrance continues to provide comfort to those affected. Developing unique ways to continue supporting people has proven to be successful. Work is ongoing. We are eager to innovate and continue providing support in these difficult times.

\section{EFFECTIVENESS OF A PALLIATIVE CARE RESOURCE TOOLKIT FOR COVID 19 FOR LOW AND MIDDLE INCOME COUNTRIES (LMICS) ON HEALTH CARE WORKERS KNOWLEDGE AND CONFIDENCE LEVELS}

Sunitha Daniel, Chitra Venkateswaran, Rajashree K Chittazhathu, Smriti Rana, Mhoira Leng, PalliCovidKerala Study Group. National Health Mission, General Hospital Ernakulam, Kochi, India Believers Church Medical College, Thiruvalla, Kerala, India Amrita Institute of Medical Sciences, Amrita University, Kochi, India Wolfson Palliative Care Research Centre, University of Hull

\subsection{6/spcare-2021-PCC.49}

Background Integrated palliative care (PC) has an important role in supporting those affected by the global COVID 19 pandemic. Communication and goals of care, symptom control and holistic support is needed particularly for patients and families living with multi-morbidity and populations in isolation and lockdown. Equipping health care workers (HCW) with core PC competencies is essential and often lacking. Building on models of integrated PC and effective response to humanitarian emergencies in Kerala, we developed and disseminated a Palliative Care in COVID-19 Resource Toolkit for LMICs comprising an e-book, webinars and ECHO platform interactive sessions for HCW.

Objective To evaluate the impact of the Resource Toolkit on the knowledge and confidence levels of HCWs.

Methods Participants registered for training package completed a pre and post course questionnaire with eighty percent attendance along with giving narrative feedback. Data collected from June to September 2020.

Results A total of 388 participants from 8 countries including 24 Indian states; 27\% male; median age 33 (20-65); 46\% nurses \& 27\% doctors. There is statistically significant improvement in all factors assessed from pretest to post test. $(\mathrm{p}=0.000))$ Mean difference in knowledge \& confidence in communication, goal setting, physical symptoms management, distress management and EOLC are as follows: 2.57 CI 95\% ; (2.21 to 2.93), 2.34 CI 95\%; (1.99 to 2.68), 2.72 CI 95\%; (2.36 to 3.07 ), 2.55 CI 95\%; (2.20 to 2.90), 2.42 CI 95\%; (2.05 to 2.79), 2.38 CI 95\%; (2.01 to 2.75), 2.88 CI 95\%; (2.51 to 3.24$), 2.63$ CI $95 \%$; (2.27 to 2.99$)$, 3.01 CI $95 \%$; (2.65 to 3.38$), 2.76$ CI $95 \%$ (2.39 to 3.13 ).

Conclusion The Toolkit when combined with online interaction can support the integration of PC competencies in health care workers many of whom have no previous exposure to PC. Narrative feedback also supported the place of a safe forum to share. Further study is planned to assess the educational impact on practice.

Funding Self-funded.

\section{VIEWING THE DECEASED PERSON: BEREAVED FAMILY UPTAKE OF A NEW SERVICE IN THE WAKE OF COVID- 19 RESTRICTIONS}

Wendy M Walker, Ruth Horton, Jenny Jones, Julie Morrell, Elaine Roberts. The Royal Wolverhampton NHS Trust

\subsection{6/spcare-2021-PCC.50}

Background Care after death includes supporting the bereaved. Evidence suggests that opportunity to view the deceased person is helpful to grieving families. During the COVID-19 pandemic, imposed restrictions to family presence challenged 
viewing arrangements. Some funeral firms also had essential health and safety policies in place which restricted visiting in a Chapel of Rest.

Methods To help meet the needs of grieving families whose relative died in an acute hospital setting, bereavement, chaplaincy and mortuary teams, together with essential administrative, portering and housekeeping staff implemented a service of COVID-secure supported viewing. Viewing the deceased person was facilitated in accordance with the family preference to be accompanied by the hospital bereavement nurse or by a male or female member of the hospital chaplaincy team of a specific faith, and included the option of prayer with or without family presence. Each family was offered a prayer booklet which was personalised with the name of the deceased. This was presented at the end of the viewing or sent by post.

Results In the period 14th April to 31st July 2020, 560 families were offered the service, and the uptake was 404 (72\%). Of the 404 families:

- Just over half $(51 \%, \mathrm{n}=205)$ required accompanied viewings, of which:

- $128(62.4 \%)$ requested/received prayer/faith-based support.

○ $77(37.6 \%)$ requested/received bereavement nurse support only.

- The majority $(81 \%, \mathrm{n}=327)$ opted for chaplaincy team involvement. This comprised:

- $128(39.1 \%)$ viewings with prayer.

- 199 (60.9\%) prayers without the family present.

- A total of 840 personalised prayer booklets were produced. Several families requested and received multiple copies.

Conclusion Our supported viewing service serves as an example of the extraordinary caring creativity in the wake of COVID-19. It has been a real privilege to create something very precious and meaningful at such a challenging time in peoples' lives.

\section{Poster 33 | dementia}

\section{FAMILY CARERS AND PROFESSIONALS' EXPERIENCES OF MANAGING PEOPLE LIVING WITH DEMENTIA - NUTRITION AND HYDRATION NEEDS TOWARDS THE END-OF-LIFE}

${ }^{1}$ Yolanda Barrado-Martín, ${ }^{1}$ Pushpa Nair, ${ }^{2,3}$ Kanthee Anantapong, ${ }^{1}$ Narin Aker, ${ }^{2,4}$ Kirsten J Moore, ${ }^{5}$ Christina H Smith, ${ }^{1}$ Greta Rait, ${ }^{2,6}$ Elizabeth L Sampson, ${ }^{7}$ Jill Manthorpe, ${ }^{1,2}$ Nathan Davies. ${ }^{1}$ Research Department of Primary Care and Population Health, UCL; ${ }^{2}$ Marie Curie Palliative Care Research Department, UCL; ${ }^{3}$ Department of Psychiatry, Prince of Songkla University; ${ }^{4}$ National Ageing Research Institute, Parkville, Victoria, Australia; ${ }^{5}$ Language and Cognition, Division of Psychology and Language Sciences, UCL; ${ }^{6}$ Barnet Enfield and Haringey Mental Health Trust Liaison Team, North Middlesex University Hospital; ${ }^{7}$ NIHR Policy Research Unit in Health and Social Care Workforce Research Unit, and NIHR Applied Research Collaborative (ARC) South London, King's College London

\subsection{6/spcare-2021-PCC.5}

Background People living with dementia may experience difficulties with eating and drinking, affecting their nutrition and hydration throughout the dementia trajectory. Such difficulties increase towards the end-of-life causing a strain on family carers. The aim of this study was to understand the needs of family carers and professionals supporting the person with dementia with nutrition and hydration towards the end-of-life and the strategies used to manage these challenges.

Methods Forty-one semi-structured interviews with family carers $(n=21)$ and professionals $(n=20)$ were conducted in London and surrounding areas in 2019-20 to explore their experiences. Interviews were audio-recorded, transcribed verbatim and analysed using thematic analysis methods.

Results Five themes were identified: family carers struggling before seeking help, perceived priorities of care, professionals' support and educational roles, nutrition and hydration challenges, and strategies. Family carers often find it hard to adapt to the changing nutrition and hydration challenges associated with the progression of dementia. Comfort becomes the focus of care instead of nutrition once the approach of the end of life is accepted. Professionals and family carers need to work together to better respond to changing needs and may be able to learn from one another. Physical impairments also impact on initial cognitive difficulties affecting individuals' nutrition and hydration needs towards the end-of-life. Flexibility and creativity are key to adapt to these changing needs towards the end-of-life.

Conclusions We need to establish how and when is best to hold discussions with family carers about changes in nutrition and hydration associated with the progression of dementia. Professional support to understand the transition towards care provision that becomes more focused on ensuring comfort and enjoyment towards the end-of-life may be helpful to family carers. The components of this and its acceptability need to be explored as well as professional capability and capacity.

\section{Posters $34-56$ | education and training}

\section{INSPIRING THE FUTURE GENERATION OF ONCOLOGISTS: A UK-WIDE STUDY OF MEDICAL STUDENTS' VIEWS TOWARDS ONCOLOGY}

Kathrine Rallis, Anna Maria Wozniak, Sara Hui, Marios Nicolaides, Neha Shah, Beena Subba, Apostolos Papalois, Michail Sideris. Barts and The London School of Medicine and Dentistry, Queen Mary University of London; Newham University Hospital, Barts Health NHS Trust, London; North Middlesex University Hospital NHS Trust, London; Experimental Educational and Research Centre ELPEN, Athens, Greece; Women's Health Research Unit, Queen Mary University of London

\subsection{6/spcare-2021-PCC.52}

Background One in 2 people born in the UK after 1960 are expected to require oncology input in their lifetime. However, only $36 \%$ of UK medical schools provide dedicated oncology placements and teaching indicating a discordance between public health impact and training. We designed a UK-wide survey to capture medical students' views on current oncology teaching and the potential role of a national undergraduate oncology symposium as an educational, networking and motivational tool.

Methods We undertook a national cross-sectional survey of UK medical students' views in oncology and satisfaction with teaching using pre-designed questionnaires. We also distributed a dedicated survey (pre and post-conference) to compare medical students' motivation towards a career in oncology after attending the national symposium. This study was prospectively approved by QMUL Ethics Committee (Reference number QMREC2348). Statistical analysis included univariate inferential tests on SPSS and GraphPad software. 